\title{
PHOTOINDUCED ELECTRON TRANSFER IN LANGMUIR-BLODGETT FILMS
}

\author{
RAMESH C. AHUJA AND DIETMAR MÖBIUS \\ Max-Planck-Institut für Biophysikalische Chemie, Am Faßberg, 3400 Göttingen (F.R.G.) \\ (Received April 25, 1989; accepted May 29, 1989)
}

Photoinduced electron transfer from an excited donor molecule (oxacyanine) to an acceptor molecule (viologen) located at the same interface in monolayer assemblies has been studied by measuring steady state fluorescence quenching and the excited state decay of the donor molecule. Donor and acceptor molecules are incorporated in matrix monolayers of arachidic acid and methyl arachidate, with a molar ratio 9:1. It is seen that the electron transfer efficiency increases with increasing donor density $\left(\sigma_{d}=0.005-0.425 \mathrm{~nm}^{-2}\right)$. The fluorescence decay functions of the donor are described by assuming at least two fluorescent species and the results show that the contribution of long-lived species increases with increasing donor density. The average rate constant for the excited state electron transfer depends on the donor density and was found to be $2 \times 10^{8} \mathrm{~s}^{-1}\left(\sigma_{\mathrm{a}}=0.025 \mathrm{~nm}^{-2}\right.$; $\sigma_{\mathrm{d}}=0.005 \mathrm{~nm}^{-2}$ ). The results are rationalized in terms of energy delocalization via incoherent exciton hopping.

\section{INTRODUCTION}

The photoinduced electron transfer between an excited donor molecule and an electron acceptor molecule is a fundamental process of great significance in many areas of physics, chemistry and biology ${ }^{1-3}$. This phenomenon has been investigated in various systems from the point of view of elucidating the mechanism and various parameters involved in the electron transfer process ${ }^{4-6}$. The important parameters are the distance between the donor and acceptor molecules, symmetry and energetic match of molecular orbitals involved, and the polarity of the medium. We have investigated the photoinduced electron transfer process in molecular organizates based on the Langmuir-Blodgett technique. These organizates have the advantage that the molecules are fixed in a rigid structure and the distance between the molecular planes is accurately controllable. In addition, the donor and acceptor molecules may be located at the same interface in the assembly but be incorporated in separate mixed monolayers. This allows for independent variation of the donor and acceptor molecules in the matrix monolayers. An excited donor molecule has a certain probability to transfer the electron to an acceptor molecule. The excited 
donor molecule may also transfer its energy to its nearest neighbour if there are other donor molecules in the vicinity, which may then transfer the electron to a nearby acceptor molecule. This leads to an enhancement in the electron transfer yield and it is this phenomenon that is the focus of the present report.

\section{EXPERIMENTAL DETAILS}

Arachidic acid (AA) and methyl arachidate (MA) were obtained from E. Merck. The acid was recrystallized from ethanol and the ester was used as received. The $N, N^{\prime}-$ dioctadecyloxacyanine perchlorate (the donor (D)) and $N, N^{\prime}$-dioctadecyl viologen perchlorate (the acceptor (A)) were synthesised by the method of Sondermann ${ }^{7}$. The monolayers were formed by spreading a chloroform solution of donor or acceptor and the matrix molecules on the aqueous (Millipore water) subphase containing $0.3 \mathrm{mM} \mathrm{CdCl}_{2}$ and $0.05 \mathrm{mM} \mathrm{NaHCO}_{3}$. The matrix for active components was a mixture of AA and MA, the molar ratio being 9:1. In addition, hexadecane (HD) was added to the spreading solution to facilitate homogeneous mixing of components in the monolayer. The monolayer was held at $40 \mathrm{mN} \mathrm{m}^{-1}$ for $20 \mathrm{~min}$ before being transferred to the quartz substrate. The system is shown schematically in Fig. 1. The monolayer-coated substrate was divided in two parts. In the reference section, the

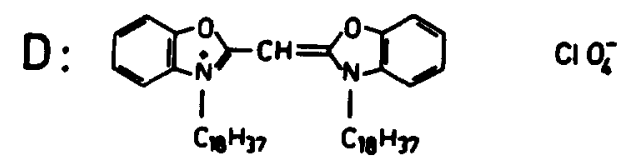
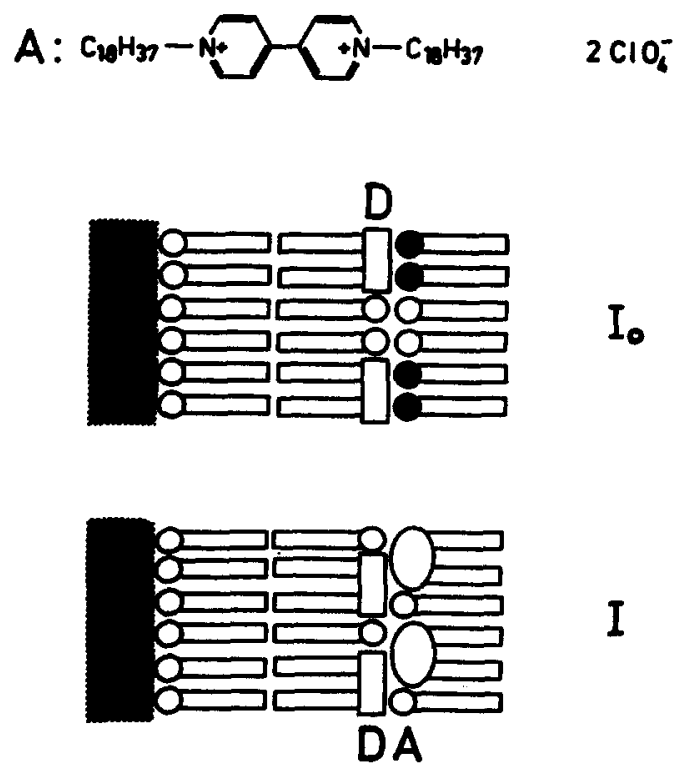

Fig. 1. Schematic diagram of the monolayer assemblies used for photoinduced electron transfer investigations. The matrix molecules are AA and MA. In the reference section $\left(I_{0}\right)$ the acceptor molecules are replaced by eicosylammonium ions. 
acceptor molecules were replaced by eicosylammonium ions in order to match the density of positive charges in the acceptor section of the substrate. The density of donor molecules in the monolayer (adjusted by varying the molar fraction of donor molecules in the monolayer) was calculated from the molecular areas $\left(0.195 \mathrm{~nm}^{2}\right.$ for the matrix molecule and $0.4 \mathrm{~nm}^{2}$ for the donor molecule). The techniques used for investigating the photoinduced electron transfer process were steady state fluorescence quenching and the measurement of the excited state lifetime of the donor molecule. The relative fluorescence intensity ratio $I / I_{0}$ (measured at $420 \mathrm{~nm}$ with excitation at $366 \mathrm{~nm}$ ) was determined by averaging the intensity in sections of the substrate with and without the acceptor monolayer. Fluorescence decay functions were measured by the correlated single-photon counting technique using a nanosecond flash lamp. The excited state lifetime was determined by convoluting model functions with the instrument response functions and fitting these with the measured fluorescence decay functions using the non-linear least-squares fitting technique.

\section{RESULTS}

The transmission (full line) and fluorescence (broken line) spectra of the mixed donor monolayer at two different donor densities $\left(\sigma_{\mathrm{d}}=0.01 \mathrm{~nm}^{-2}\right.$, curve a; $\sigma_{\mathrm{d}}=0.425 \mathrm{~nm}^{-2}$, curve b) are shown in Fig. 2. It is seen that, as the donor density increases, the fluorescence maximum shifts towards the red by $c a .15 \mathrm{~nm}$ and also the ratio of monomer to dimer changes. The fluorescence of the donor monolayer (monitored at $420 \mathrm{~nm}$ ) is partly quenched in the presence of the acceptor in the adjacent monolayer (molar ratio A:MA:AA:HD = 1:20:180:200; $\sigma_{\mathrm{a}}=0.025 \mathrm{~nm}^{-2}$ ). The relative fluorescent intensity ratio $I / I_{0}$ of the donor monolayer depends on the donor density as shown in Fig. 3. The experimental results are indicated by asterisks. It is seen that the ratio $I / I_{0}$ decreases with increasing donor density $\sigma_{\mathrm{d}}$ and approaches a limiting value for large $\sigma_{\mathrm{d}}$. Figure 4 shows the fluorescence decay functions of the mixed donor monolayer (at various donor densities) together with the time profile of the excitation source (curve a).

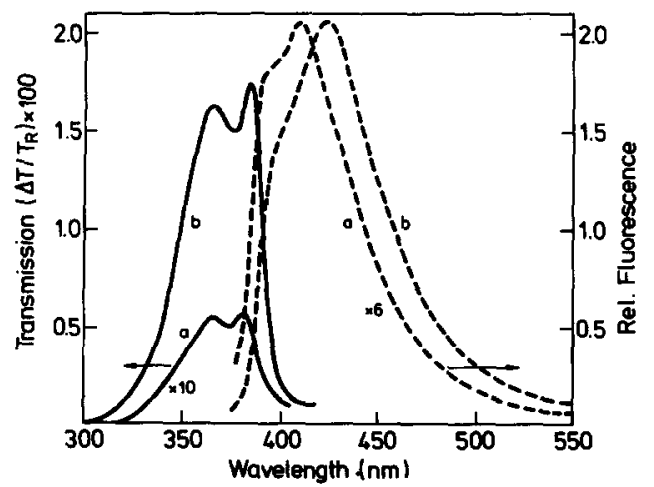

Fig. 2. Transmission (- - and fluorescence (--) spectra of the donor monolayer at two donor densities: curve a, $\sigma_{\mathrm{d}}=0.01 \mathrm{~nm}^{-2}$; curve $\mathrm{b}, \sigma_{\mathrm{d}}=0.425 \mathrm{~nm}^{-2}$. The excitation wavelength for emission is $366 \mathrm{~nm}$. 


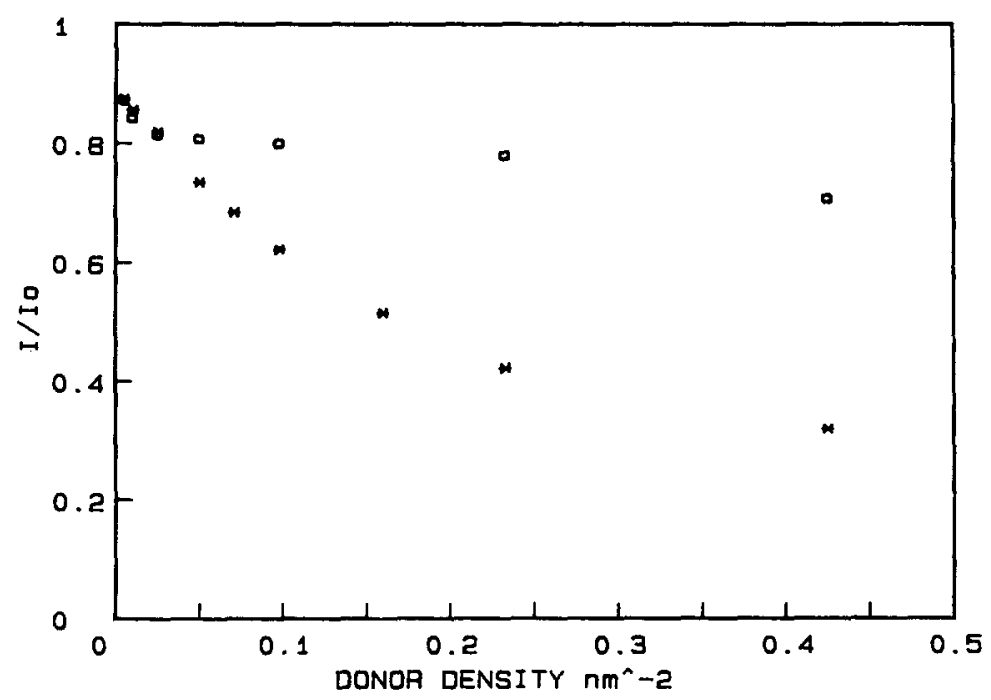

Fig. 3. Relative donor fluorescent intensities $I / I_{0}$ as a function of donor density $\sigma_{\mathrm{d}}\left({ }^{*}\right)$ at constant acceptor density $\sigma_{\mathrm{a}}=0.0254 \mathrm{~nm}^{-2}$. Values of $I / I_{0}$ are calculated from the excited state lifetime data (0).

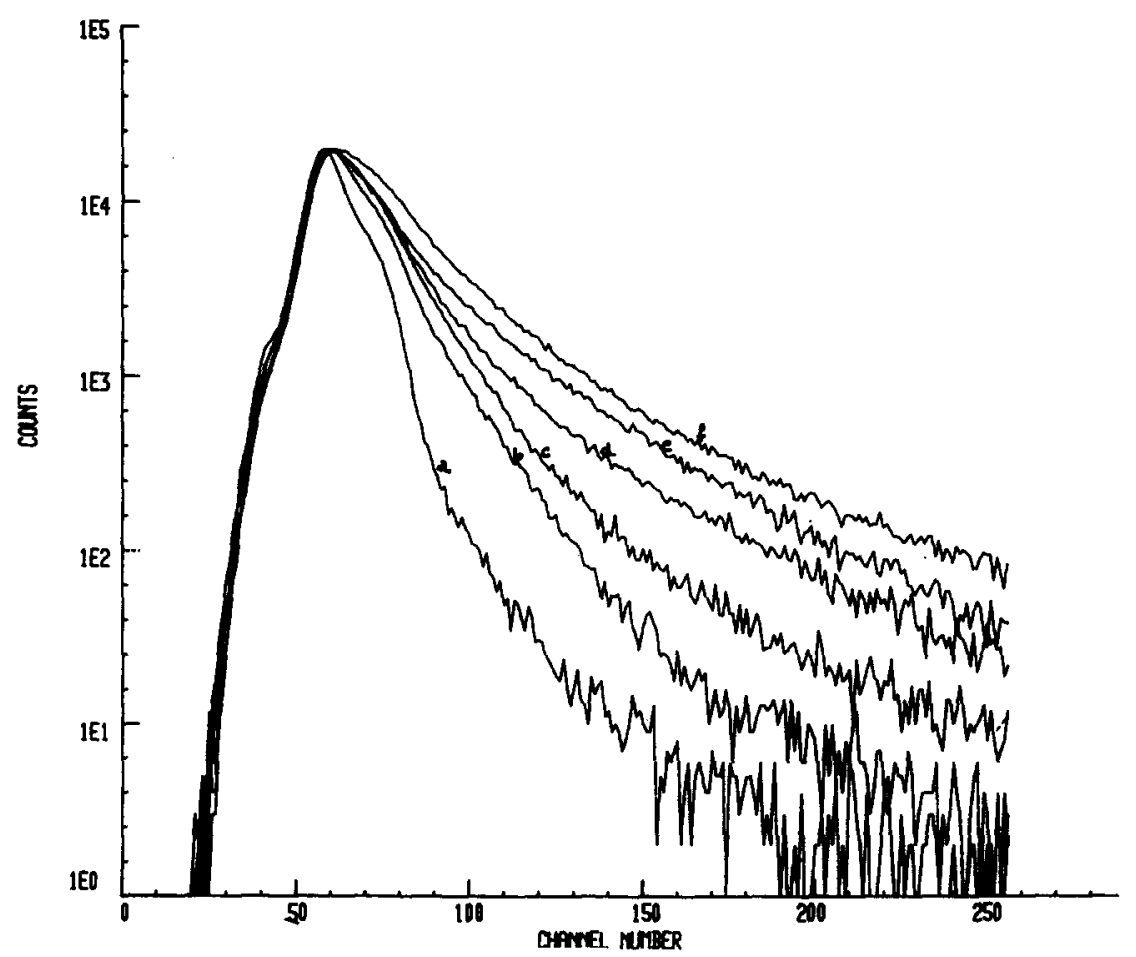

Fig. 4. Fluorescence decay functions of the donor at various donor densities (curves $b-f$ ) and the time profile of the excitation source (curve a): the donor densities $\sigma_{\mathrm{d}}$ are $0.005 \mathrm{~nm}^{-2}$ (curve b), $0.025 \mathrm{~nm}^{-2}$ (curve c), $0.05 \mathrm{~nm}^{-2}$ (curve d), $0.098 \mathrm{~nm}^{-2}$ (curve e) and $0.425 \mathrm{~nm}^{-2}$ (curve f); the time scale is $0.125 \mathrm{~ns}$ per channel. 
These results have been analysed by assuming two fluorescent species in the donor monolayer. It is seen that, as the donor density in the monolayer is increased, the contribution of the long-lived species to the fluorescence decay also increases.

\section{DISCUSSION}

The results presented in Fig. 3 show that the fluorescence quenching and thereby the yield and efficiency of photoinduced electron transfer increase with increasing donor density. Similar results have been interpreted in the past ${ }^{8}$ in terms of energy delocalization via incoherent exciton hopping in the donor monolayer. This interpretation is, however, only valid if the association behaviour of the donor molecule does not change with increasing donor density. That this is not the case is seen from the changes in the fluorescence decay functions ( $c f$. Fig. 4) with increasing donor density. The decay functions cannot be described by a single exponential (monomer) and at least two exponentials (two species) are needed. The decay function analysis shows that, in contrast to the usual behaviour, the contribution of the long-lived species increases with increasing donor density. This may be due to phase separation and/or association of molecules to dimers or $\mathrm{H}$ aggregates, which is known to occur both in the solution and in monolayer assemblies ${ }^{9}$. The evidence for the association of oxacyanine dye (donor) in the mixed monolayer at higher donor densities is also provided by the fluorescence and absorption spectra data (cf. Fig. 2). An increasingly dominant absorption band $(366 \mathrm{~nm})$ shifted to shorter wavelengths with respect to the monomer band $(385 \mathrm{~nm})$ is found for monolayers of donor densities $\sigma_{\mathrm{d}} \geqslant 0.02 \mathrm{~nm}^{-2}$. Further, the fluorescence maximum shifts from $410 \mathrm{~nm}\left(\sigma_{\mathrm{d}}=0.005 \mathrm{~nm}^{-2}\right)$ to $425 \mathrm{~nm}\left(\sigma_{\mathrm{d}}=0.425 \mathrm{~nm}^{-2}\right)$. In addition, the timeresolved fluorescence spectroscopic data ${ }^{10}$ (change in Stokes' shift with increasing donor density) indicate that the Stokes shift is changed as a step function at the threshold time depending on the donor density. The spectral data at any time can be approximated by a sum of monomer and dimer spectra. Assuming a single rate constant $k_{\mathrm{et}}$ for the electron transfer for both the species, $k_{\mathrm{et}}=2 \times 10^{8} \mathrm{~s}^{-1}$ has been calculated from the value of $I / I_{0}=0.875$ and the evaluated lifetime and amplitude data (species $1,0.176 \mathrm{~ns}(57 \%)$; species $2,1.36 \mathrm{~ns}(43 \%)$ of the fluorescence decay curve (Fig. 4, curve b). The calculated values of $I / I_{0}$ with this rate constant for the other donor densities are shown in Fig. 4 (circles). Thus, although these data demonstrate an increase in electron transfer efficiency with increasing donor density, it is to a much smaller extent than the observed data. We conclude therefore that, to rationalize the experimental data, energy delocalization via incoherent exciton hopping has still to be invoked. A similar conclusion is also reached by Tamai et al. ${ }^{10}$ who think that the excitation energy migrates among monomer sites with similar energy levels before being trapped at dimers. A detailed model which takes this into account together with the other results on photoinduced electron transfer obtained by us is under development and will be reported in the near future.

\section{CONCLUSIONS}

It is observed that the yield of electron transfer from an excited donor to an 
acceptor located at the same interface in monolayer assemblies increases with increasing donor density (light harvesting effect). In addition, the association behaviour of the donor molecules in the matrix monolayer changes (increasing contribution of long-lived fluorescent species) with increasing donor density. This, however, is not sufficient to explain the observed increase in electron transfer yield and energy delocalization via incoherent exciton hopping still has to be invoked to rationalize the results.

\section{REFERENCES}

1 D. Möbius, Mol. Cryst. Liq. Cryst., 96 (1983) 319.

2 H. Kuhn, in H. Gerischer and J. J. Katz (eds.), Light-Induced Charge Separation in Biology and Chemistry, Verlag Chemie, 1979, p. 151.

3 M. Calvin, Photochem. Photobiol., 23 (1976) 425.

4 H. Kuhn, Pure Appl. Chem., 53 (1981) 2105.

5 K. P. Seefeld, D. Möbius and H. Kuhn, Helv. Chim. Acta, 60 (1977) 2608.

6 D. Möbius, in J. Rabani (ed.), Photochemical Conversion and Storage of Solar Energy, Weizmann Science Press, 1982, p. 139.

7 J. Sondermann, Liebigs Ann. Chem., 749 (1971) 183.

8 D. Möbius, in G. R. Freeman (ed.), Kinetics of Nonhomogeneous Processes, Wiley, New York, 1987, p. 533.

9 D. Möbius, Ber. Bunsenges. Phys. Chem., 82 (1978) 848.

10 N. Tamai, T. Yamazaki and I. Yamazaki, Proc. 4th Int. Conf. on Langmuir-Blodgett Films, Tsukuba, April 1989, in Thin Solid Films, 179 (1989) 451. 